International Journal Of Advanced Smart Sensor Network Systems ( IJASSN ), Vol 2, No.2, April 2012

\title{
ELITIST GENETIC ALgORITHM BASED ENERGY EFFICIENT ROUTING SCHEME FOR WIRELESS SENSOR NETWORKS
}

\author{
Vinay Kumar Singh ${ }^{1}$, Vidushi Sharma ${ }^{2}$ \\ ${ }^{1}$ Department of Computer Science \& Engineering and Information Technology, \\ Anand Engineering College, Agra, India, \\ vksingh100@rediffmail.com \\ ${ }^{2}$ School of Information \& Communication Technology, Gautam Buddha University, \\ Greater Noida, India, \\ vidushi@gbu.ac.in
}

\begin{abstract}
Wireless Sensor Networks have gained world-wide attention in recent years due to the advances made in wireless communication, information technologies and electronics field. They consist of resource constrained sensor nodes that are usually randomly or manually deployed in an area to be observed, collecting data from the sensor field and transmitting the gathered data to a distant Base Station. The nodes are energy limited sensors, and therefore it is important to increase the network lifetime. Energy saving is one of the critical issues in the routing design in WSNs. The factors causing the unequal energy dissipation are firstly, the distance between the nodes and base station and secondly, the distance between the nodes themselves. Using traditional methods it is difficult to obtain the high precision of solution as the problem is NP hard. Applying genetic algorithms (GAs) in finding energy efficient shortest route for WSNs is emerging as an important field. The routing in WSN is a combinatorial optimization problem, hence GA can provide optimized solution to energy efficient shortest path problem in WSN. This paper uses a forward address based shortest path routing in the network. Genetic algorithm with elitism concept is used to obtain energy efficient routing by minimizing the path length and thus maximizing the life of the network. The proposed algorithm has its inherent advantage that it keeps the elite solutions in the next generation so as to quickly converge towards the global optima. The results show that GAs are efficient for finding the optimal energy constrained route as they can converge faster than other traditional methods used for combinatorial optimization problems.
\end{abstract}

\section{KEYWORDS}

Energy Efficient, Genetic Algorithm, Elitism, Wireless Sensor Networks

\section{INTRODUCTION}

A Wireless sensor network consists of randomly/manually deployed sensors that sense the physical or environmental events and send the data to the base station. A large number of small, inexpensive, disposable and autonomous sensor nodes are generally deployed in an ad hoc manner in vast geographical areas for remote operations. Sensor nodes in a WSN are constrained in storage capacity, computation power, bandwidth and power supply[1, 2, 3]. The development

DOI: $10.5121 /$ ijassn.2012.2202 
International Journal Of Advanced Smart Sensor Network Systems ( IJASSN ), Vol 2, No.2, April 2012

of low-cost, low-power, a multifunctional sensor has received increasing attention from various industries. Sensor nodes are smaller in size and capable of sensing, gathering and processing data. They also communicate with other nodes in the network, via radio frequency (RF) channel. The areas of applications of WSNs vary from civil, healthcare, and environmental to military[4].

Energy Efficient Routing in Wireless sensor networks has gained a lot of attraction from the researchers in the recent years. In [5, 6] summary of recent research results on energy efficient data routing in sensor networks is given. To increase the network lifetime, the design of efficient routing protocol for communication is very important. An illustration of the existing routing protocol for wireless sensor network using data centric approach and present performance analysis of these protocols is done in [7]. Evolutionary Algorithms can be used effectively to find the energy efficient path in wireless sensor networks [8]. A simple approach to minimize the average path length is proposed in [9] where they considered the wireless network of transceiver nodes with a known spatial distribution using a Genetic Algorithm optimization method. Each of the nodes consists of a relatively simple a transceiver (antennas, a receiver and a transmitter). The goal of the optimization is to minimize the average path length from source to destination to minimize the transmitted power. Further, a method proposed in [10] has used a multipath routing protocol for wireless sensor networks that enhances the reliability of WSN by using multipath routing. It is useful for delivering data in unreliable environments. The idea is to define many paths from source to sink and send through them the same subpackets. This implies that the traffic will increase significantly (not energy aware), but increasing the reliability of the network. The idea is to split the original data packet into subpackets through each path. This can offer at the end, even with the loss of subpackets, the reconstruction of the original message. Energy aware consideration is not done which may reduce the lifetime of the sensor network. The energy awareness in multi path routing is done in [11-14] with consideration of maximum lifetime routing in Wireless Sensor Networks. This protocol routes data through a path whose nodes have the largest residual energy. The path is switched whenever a better path is discovered. The primary path will be used until its energy is below the energy of the backup path. By means of this approach, the nodes in the primary route will not exhaust their energy by the continual use of the same path, thus achieving longer lifetime.

Recent advances in WSN have led to searching for new routing schemes for wireless sensors where energy awareness is essential consideration [15]. The aim of networks is to achieve high quality of service (QoS) communication, thus sensor network schemes must focus primarily on power conservation. Ad hoc routing techniques proposed in the literature do not usually fit the requirements of the sensor networks. Hence, it is necessary to design a special multi hop wireless routing technique between the sensor nodes and the sink node with a focus on energy efficiency. A clustering method is proposed in [16] where a fixed number of cluster heads are selected based on maximum residual energy of the nodes. Each cluster head is associated with a group of nodes based on the minimum distance among them. In such scheduling, all the nodes dissipate uniform energy and subsequently remain alive for long time.

Rest of the paper is organized as follows: Section 2 describes the network model used in this paper, section 3 talks about the modified GA approach with elitism used for WSN routing, section 4 provides and analysis of the results obtained using the proposed scheme and section 5 is the conclusion.

\section{THE Proposed MODEL}

The model considered in this paper realizes a powerful Base Station which has adequate source of energy supply and it is located far away from the sink. The sensor nodes are homogeneous and have limited energy. All the nodes have uniform initial energy allocation. The first order radio 
model is used for calculation of the energy dissipation for data communication operations like transmission and reception. The energy spent by a node in transmitting a k-bit packet to another node $\mathrm{d}$ meters away, is given by:

$\operatorname{ETX}(\mathrm{k}, \mathrm{d})=\left(\xi_{\text {elec }}+\xi_{\text {amp }} * \mathrm{~d}^{\mathrm{n}}\right) * \mathrm{k}$

and the energy spent in receiving the packet is,

$\operatorname{ETX}(\mathrm{k})=\xi_{\text {elec }} * \mathrm{k}$

The energy spent in transmitting a packet from node $i$ to $j$ is the same as that from node $j$ to $i$ for any given value of SNR i.e. there is a symmetric channel. The computational energy is negligible in comparison to communicational energy and can be ignored. A routing path or chain is an ordered sequence of the nodes in the network that transmit the data message towards the sink node. A time interval in which a node generates a packet and transmits it towards its neighbor is called a data collecting round. After receiving a packet from its previous node, every node relays it to the next node in the chain. The task is to minimize the energy dissipation in the nodes, by the formation of an optimal data collecting chain. Considering $\mathrm{N}$ nodes in the network, the total energy expended in a typical data gathering round is the summation of the energy dissipated by the individual sensor nodes. Total energy spent can be calculated for a packet size of $\mathrm{k}$ as follows,

$\mathrm{E}_{\text {TOTAL }}=\left\{\sum_{\mathrm{i}=1}^{\mathrm{N}-1}\left(\xi_{\text {elec }}+\xi_{\text {amp }} * \mathrm{~d}^{\mathrm{n}}\right)+\xi_{\text {elec }}\right\} * \mathrm{k}$

di denotes the distance between the ith node and the (i+1)th node in the data collecting chain. A threshold value is imposed on the communication radius between the nodes as dTH. This ensures reliable communication in between the nodes reducing unwanted noise and packet loss probability also this is necessary to reduce the energy consumption.

\section{IMPROVED GENETIC AlgORITHM}

Genetic Algorithms (GAs) are stochastic search mechanisms. They are mainly a probabilistic search \& optimization technique inspired by the mechanics of (Darwinian) natural selection and genetics [17]. It has been shown [18-20] that genetic algorithm performs well in solving the problem of finding path optimization for WSN. It works on a group of optimal or suboptimal solution during the search and gives the optimal search path within a short time.

The algorithm works by storing useful information about the individuals from the current population either implicitly through redundant representations [21-23].The elitist strategy ensures that the best individual will not be destroyed. Instead, the best individual will take place of the worst individual after the operations of crossover and mutation. If the energy of a particular node in the routing reaches below a predefined level, the node is replaced in the routing chain based on some probability. This is done, to prolong the lifetime of the network. Also new individuals are inserted into the population after every generation so that the diversity is maintained and GA does not get converge into local optima as shown in Figure 1. The steps of the modified GA are:

1. Population Initialization: In the first step $\mathrm{N}$ individuals (chromosomes) are produced randomly and the evolutionary generation starts with iteration 0 . The distance threshold (communication radius) is initialized. 
2. Fitness Calculation: The fitness function is designed to increase the lifetime of the network, which evaluates whether, a particular chromosome increases lifetime of the network or not. The algorithm preserves the historically obtained best chromosome, that is, with the highest fitness value this is called elitism. The fitness of each chromosome is calculated by

$$
f(C)=\sum_{i=1}^{N-1} d_{i}^{2}
$$

Above Equation calculates the energy of a chromosome $\mathrm{C}$ containing $\mathrm{N}$ genes and di denotes the distance between the (i+1)th node (or, gene) and the ith node in the data gathering chain. A greater value of the chromosome energy indicates a longer data gathering chain and which means an inferior solution.

3. Selection: This step selects the better individuals according to the corresponding selection operator. The selection operator used here is tournament selection where by a mating pool of the individuals with above average fitness value is maintained and two parents are randomly selected for crossover.

4. Crossover: This step produces the new individuals to complete the global search according to the corresponding crossover operator. Two point crossover operator is used in this paper. In two point crossover two points are randomly selected in a chromosome and all the genes from one

point to another point are copied from the first chromosome to the second chromosome.

5. Repair: This step removes the routing loop. The genes values which are repeated in the chromosome are deleted and thus the looping is removed. If a child violates the imposed constraints (distance threshold), then it is rejected and again the crossover is performed.

6. Mutation: This step produces the new individuals to maintain the diversity in the population. In mutation, a node is randomly selected from the best chromosome obtained in the past generation and a gene value is changed randomly to produce a new offspring. If the new offspring violates the constraints like distance threshold, it is rejected and mutation is performed again using repair function. The mutation operation can help the search to exit out of the local optima and search for global optima.

7. Elitism: In this step the best solutions from the population are maintained in the next generation so that the convergence is faster.

8. Checking the Terminating Criteria: If the maximum number of generations has elapsed or the energy of the network has fallen below predefined level, the algorithm is terminated.

9.Producing the Result:. The program outputs the best chromosome found so far as the result.

\section{Simulation RESUltS}

The energy dissipated per bit (in equation (3)) to run the radio electronics is $\xi e l e c(50 \mathrm{~nJ} / \mathrm{bit}$ ) and Gamp $(10 \mathrm{pJ} / \mathrm{bit} / \mathrm{m} 2)$ is the energy required by the transmit amplifier to maintain an acceptable signal to noise ratio (SNR) in order to transfer data messages reliably. $n$ is called the path loss exponent, whose value enhances with increasing channel non-linearity (usually, $2.0 \leq \mathrm{n} \leq 4.0$ ). The value of $\mathrm{n}$ is 2 for free space transmission. 
The simulations are done under the MATLAB programming environment. Custom code is written to simulate the wireless sensor network. The number of sensor nodes is varied from 10 to 50.The sensors are randomly distributed in the area of $100 \mathrm{~m} \times 100 \mathrm{~m}$. The sink node is at $(100,100)$.

The initial energy of sensor nodes is $1 \mathrm{~J}$. Every node transmits ten 4000-bits messages every round. The number of individuals in the population is the same as that of total number of nodes in the network, and the maximum number of generations for genetic algorithm is ten times as that of total number of nodes in the network.

The Figure 2 shows the comparison of the following two situations: (i) direct communication between the member node and the sink node using Simple GA (ii) using the modified elitism based genetic algorithm. The algorithms are tested for up to maximum of 50 nodes. It is observed that there is much improvement in the network lifetime using the proposed GA with elitism.

Figure (3) shows the residual energy of twenty nodes for $100 \mathrm{mx} 100 \mathrm{~m}$ network area when the first dead node appears. The average residual energy of the network using Simple GA is 0.6972J. The average energy of the network using the proposed algorithm is $0.1530 \mathrm{~J}$ which is an improvement over the other algorithms discussed. This shows that the proposed algorithm better utilizes the network energy.

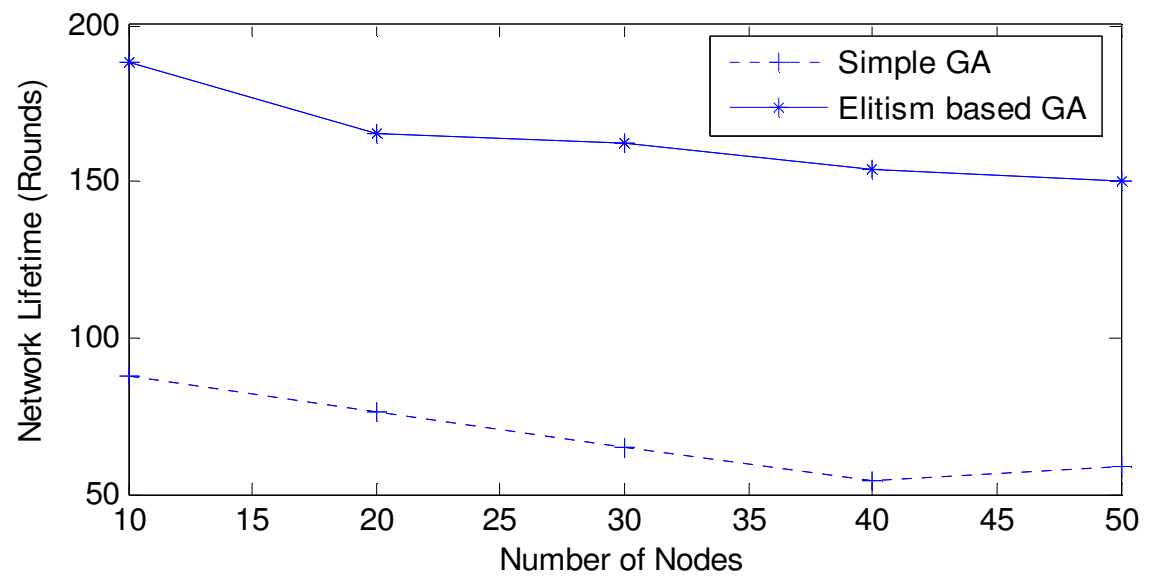

Fig 2: (a) The comparison of network lifetime under the different number of the nodes using Simple Genetic Algorithm and Elitism based Genetic Algorithm

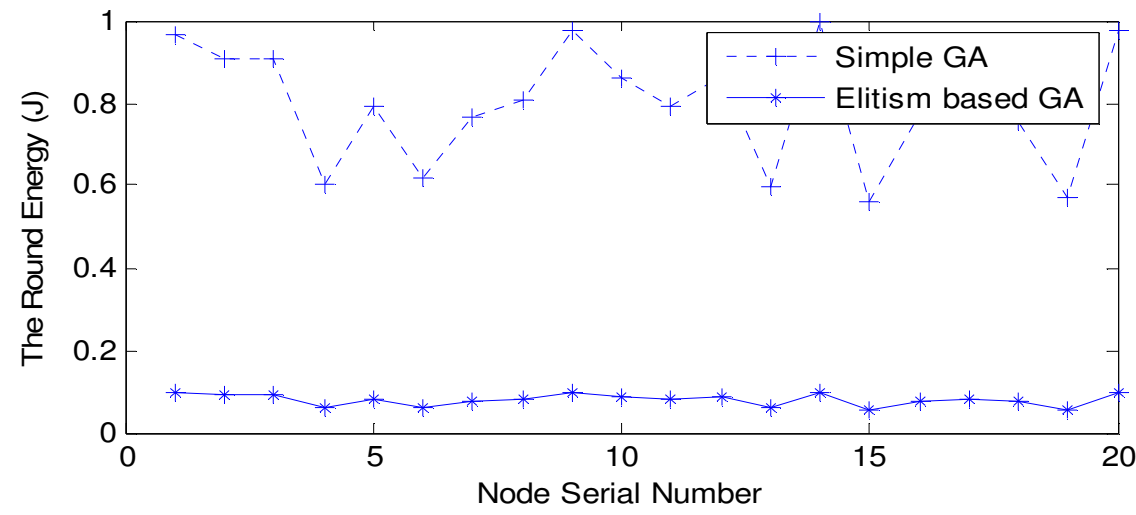

Fig 3: (a) The comparison of residual energy of the network using Simple Genetic Algorithm and Elitism based Genetic Algorithm 
International Journal Of Advanced Smart Sensor Network Systems ( IJASSN ), Vol 2, No.2, April 2012

\section{Conclusions}

In this paper, the basic genetic algorithm is improved using the elitism concept to provide the solution to combinatorial optimization problem of finding the energy efficient shortest routing for the wireless sensor networks. The modified GA uses elitist strategy so that the best individual is preserved and carried to the next generation so that there is significant improvement in the convergence. The node energy falling below predefined level is restrained from the path based on some probability to increase the overall lifetime of the network.

\section{REFERENCES}

[1] Goldberg D. E. (1989), "Genetic Algorithms in Search, Optimization, and Machine Learning" Reading, MA: Addison-Wesley.

[2] Akyildiz I. F., Su W., Sankarasubramaniam Y. (2002), Cayirci E., "A Survey On Sensor Networks," IEEE Communications Magazine, Vol. 40, Issue 8.

[3] Raghavendra V. K., Förster A. (2011), Venayagamoorthy G. K., "Computational Intelligence in Wireless Sensor Networks: A Survey,” IEEE Communications Surveys \& Tutorials, Vol. 13, No. 1, pp. 68-96.

[4] Heikalabad S. R., Ghaffari A., Hadian A., Rasouli H. (2011), "DPCC: Dynamic Predictive Congestion Control in Wireless Sensor Networks," IJCSI International Journal of Computer Science Issues, Vol. 8, Issue 1, pp.472-477.

[5] Min R., Bhardwaj M., Cho S. H. (2001), "Low Power Wireless Sensor Networks," Proceedings for International. Conference on VLSI Design, Bangalore, India ,pp.221-226.

[6] Akkaya K., Younis M. (2005), "A Survey on Routing Protocols for Wireless Sensor Networks," Elsevier Ad Hoc Networks, Vol. 3, Issue 3, pp. 325-349.

[7] Azni A. H., Saudi M. M., Azman A., Johari A. S. (2009), "Performance Analysis of Routing Protocol for WSN using Data Centric Approach," World Academy of Science, Engineering and Technology, Issue 53.

[8] Singh V. K., Sharma V. (2011), "Conference on Advancements in Communication, Computing \& Signal Processing (COMMUNE CACCS 2011)".

[9] Johnson J. M., Samii Y. R. (1995), "Genetic Algorithm Optimization of Wireless Communication Networks," Proceedings for Antennas and Propagation Society International Symposium, AP-S. Digest.

[10] Li Q., Aslam J. Rus D. (2001), "Hierarchical Power-Aware Routing in Sensor Networks," Proceedings of DIMACS Workshop on Pervasive Networking.

[11] Murthy S., Garcia-Luna-Aceves J. J. (1996), “An Efficient Routing Protocol for Wireless Networks," ACM Mobile Networks Applications”, Vol. 1, Issue 2, pp.183-197.

[12] Singh S., Woo M., Mghavendra C.S. (1998), "Power-Aware Routing in Mobile Ad Hoc Networks," ACM SIGMOBILE Mobile Computing and Communications Review,, pp. 181-190.

[13] Heinzelman W. R., Chandrakasan A., Balakrishnan H. (2000), "Energy-Efficient Communication Protocol for Wireless Microsensor Networks," Proceedings for the Hawaii International Conference on System Sciences, (IEEE), January 4-7, pp.876-882.

[14] Chang J.H., Tassiulas L. (2004), "Maximum Lifetime Routing in Wireless Sensor Networks," Journal IEEE/ACM Transactions on Networking (TON), Vol. 12 Issue 4.

[15] Yick J., Mukherjee B., Ghosal D. (2008), "Wireless Sensor Network Survey," Elsevier Computer Networks, Vol. 52, Issue 12, pp. 2292-2330.

[16] Azad P., Singh B., Sharma V. (2011), “A Novel Clustering Approach For Extending The Lifetime For Wireless Sensor Networks", International journal of Advances in Engineering \& Technology, Vol. 1 Issue $5 \mathrm{Pg}$ 441-446.

[17] Wenliang G., Huichang S., Jun Y., Yifei Z. (2009), ”Application of Genetic Algorithm in EnergyEfficient Routing" China-Japan Joint Microwave Conference, Page(s): 737 - 740.

[18] Guo L., Tang Q. (2010), “An Improved Routing Protocol in WSN with Hybrid Genetic Algorithm" Second International Conference on Networks Security Wireless Communications and Trusted Computing (NSWCTC), Vol.: 2, Page(s): 289 - 292. 
[19] Islam O., Hussain S. (2006), "An Intelligent Multi-hop Routing for Wireless Sensor Networks" International Conference on Web Intelligence and Intelligent Agent Technology Workshops, 2006. WI-IAT 2006 Workshops. 2006 IEEE/WIC/ACM, Page(s): 239 - 242.

[20] Al-Ghazal M. El-Sayed A., Kelash, H. (2007), "Routing Optimization using Genetic Algorithm in Ad Hoc Networks" The IEEE Symposium on Signal Processing and Information Technology (ISSPIT) Cairo.

[21] D. Dasgupta and D. McGregor (1992), "Nonstationary function optimization using the structured genetic algorithm," in Proc. 2nd Int. Conf. Parallel Problem Solving Nature, pp. 145-154.

[22] E. Lewis and G. Ritchie (1998), "A comparison of dominance mechanisms and simple mutation on non-stationary problems," in Proc. 5th Int. Conf. Parallel Problem Solving Nature, pp. 139-148.

[23] A. Uyar and A. Harmanci (2005), "A new population based adaptive dominance change mechanism for diploid genetic algorithms in dynamic environments," Soft Comput., vol. 9, no. 11, pp. 803-815.

\section{Authors}

Vinay Kumar Singh received his B.E.(Computer Engineering) degree from University of Pune, (Maharashtra), India in 1994 and M. Tech. degree from Dayalbagh Educational Institute, Agra (U.P) India in 2002. He is a research scholar at Gautam Buddha University, Greater Noida, (U.P.) India, and is presently working as Assistant Professor in Department of Computer Science \& Engineering and Information Technology in Anand Engineering College, Agra (U.P.) India. His research interests are Evolutionary Algorithms, Optimization and Energy Efficient Routing in Wireless Sensor Networks.

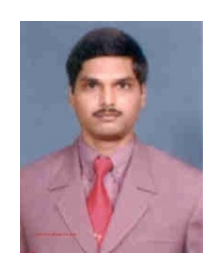

Vidushi Sharma has done Ph.D in computer Science and is presently working as Assistant Professor in Gautam Buddha University, Greater Noida (U.P.), India. She teaches post graduate and graduate level courses and has large number of International and national publications and has also written a book on Information Technology. Her research interests includes IT applications in management and performance evaluation of Information Systems which includes Wireless Systems, Application Software, Ecommerce System.

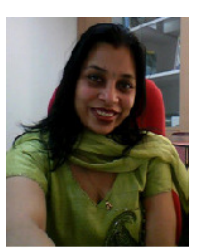

